ESAIM: COCV 19 (2013) 533-554

DOI: $10.1051 / \mathrm{cocv} / 2012020$
ESAIM: Control, Optimisation and Calculus of Variations

www.esaim-cocv.org

\title{
CONJUGATE-CUT LOCI AND INJECTIVITY DOMAINS ON TWO-SPHERES OF REVOLUTION *,**,***
}

\author{
Bernard Bonnard ${ }^{1,2}$, Jean-Baptiste Caillau ${ }^{2}$ And Gabriel Janin ${ }^{2}$
}

\begin{abstract}
In a recent article [B. Bonnard, J.-B. Caillau, R. Sinclair and M. Tanaka, Ann. Inst. Henri Poincaré, Anal. Non Linéaire 26 (2009) 1081-1098], we relate the computation of the conjugate and cut loci of a family of metrics on two-spheres of revolution whose polar form is $g=\mathrm{d} \varphi^{2}+m(\varphi) \mathrm{d} \theta^{2}$ to the period mapping of the $\varphi$-variable. One purpose of this article is to use this relation to evaluate the cut and conjugate loci for a family of metrics arising as a deformation of the round sphere and to determine the convexity properties of the injectivity domains of such metrics. These properties have applications in optimal control of space and quantum mechanics, and in optimal transport.
\end{abstract}

Mathematics Subject Classification. 58B20, 49K15, 53C22.

Received February 28, 2011. Revised November 24, 2011.

Published online February 21, 2013.

\section{INTRODUCTION}

On a Riemannian manifold, the cut point along a geodesic $\gamma$ emanating from $q_{0}$ is the first point where it ceases to be minimizing, while the first conjugate point is the first point where it ceases to be minimizing among the geodesics $C^{1}$-close to $\gamma$. Considering all the geodesics starting from $q_{0}$ they will form respectively the cut and the conjugate and cut locus. The structure of the conjugate and cut loci on a real analytic surface homeomorphic to the sphere is known after the works of Poincaré [21] and Myers [20]: the cut locus is a finite tree and the extremity of each branch is a cusp point of the conjugate locus. But the explicit computation of the branches is a very complicated problem [3] and it was proved only recently that the cut locus of any point on an ellipsoid is a segment and the conjugate locus of a non umbilical point has exactly four cusps [17]. In parallel the conjugate and cut loci were investigated for a metric $g=\mathrm{d} \varphi^{2}+m(\varphi) \mathrm{d} \theta^{2}$ on a two-sphere of revolution, when it is reflective symmetric with respect to the equator $m(\pi-\varphi)=m(\varphi)$. In particular it was shown that the cut locus is a single branch if the Gaussian curvature is monotone from the north pole to the equator [23], which generalizes the ellipsoid study and gives an important relation with curvature. Very recently, this result

\footnotetext{
Keywords and phrases. Conjugate and cut loci, injectivity domain, optimal control, optimal transport.

* Work supported by ANR Geometric Control Methods (project No. NT09_504490).

** Also supported by Conseil Régional de Bourgogne (contract No. 2009-160E-160-CE-160T) and SADCO Initial Training Network (FP7 grant No. 264735-SADCO).

*** Also supported by a DGA grant.

1 INRIA, 2004 route des lucioles, 06902 Sophia Antipolis, France. bernard.bonnard@u-bourgogne.fr

2 Institut de Mathématiques de Bourgogne, 9 avenue Savary, 21078 Dijon, France. jean-baptiste.caillau@u-bourgogne.fr; gabriel.janin@u-bourgogne.fr
} 
was improved relating the simple structure of the cut locus to the period mapping of the $\varphi$-variable [8], this extension being motivated by a family of metrics arising in space mechanics and quantum control.

Indeed besides the geometric interest of such studies, the computation of the cut locus is an important task in optimal control in the goal of computing the synthesis of the problem which relies on determining the switching and the cut loci [5] and Riemannian or sub-Riemannian metrics arise through averaging procedures. A first and important example concerns the orbital transfer between two coplanar orbits using low propulsion [7]. Considering the energy minimization problem and averaging the underlying Hamiltonian with respect to the longitude leads to the 3D-Hamiltonian:

$$
H=\frac{9 n^{1 / 3}}{2} p_{n}^{2}+\frac{1}{2 n^{5 / 3}}\left[\frac{5\left(1-e^{2}\right)}{2} p_{e}^{2}+\frac{\left(5-4 e^{2}\right)}{2 e^{2}} p_{\theta}^{2}\right]
$$

where $n$ is the mean motion, $e$ the eccentricity of the orbit and $\theta$ the angle of the pericenter, $p=\left(p_{n}, p_{e}, p_{\theta}\right)$ being the adjoint vector. Setting $n=(5 r / 2)^{6 / 5}$ and $e=\sin \varphi, H$ is the Hamiltonian associated with the Riemannian metric

$$
\mathrm{d} s^{2}=\mathrm{d} r^{2}+\frac{r^{2}}{c^{2}}\left(\mathrm{~d} \varphi^{2}+\frac{\sin ^{2} \varphi}{1-\left(1-\mu^{2}\right) \sin ^{2} \varphi} \mathrm{d} \theta^{2}\right)
$$

with $c=\sqrt{2 / 5}$ and $\mu=1 / \sqrt{5}$. By homogeneity one can restrict to $\mathrm{d} r=0$ and this leads to the metric: $\mathrm{d} \varphi^{2}+m(\varphi) \mathrm{d} \theta^{2}$

$$
m(\varphi)=\frac{\sin ^{2} \varphi}{1-\left(1-\mu^{2}\right) \sin ^{2} \varphi}
$$

describing the evolution of the $\varphi$-variable. As $e$ belongs to $[0,1]$, such a metric is defined in the north hemisphere but can be extended analytically to the whole sphere. It is a deformation of the round sphere through the homotopy

$$
m_{\lambda}(\varphi)=\frac{\sin ^{2} \varphi}{1-\lambda \sin ^{2} \varphi}
$$

and the case $\lambda=1$ defines an almost-Riemannian metric with a polar singularity at the equator. If we suppose that the thrust is oriented only in the tangential direction, the Hamiltonian becomes

$$
H=\frac{9 n^{1 / 3}}{2} p_{n}^{2}+\frac{1}{2 n^{5 / 3}}\left[\frac{4\left(1-e^{2}\right)^{3 / 2}}{1+\sqrt{1-e^{2}}} p_{e}^{2}+\frac{4\left(1-e^{2}\right)}{1+\sqrt{1-e^{2}}} \frac{p_{\theta}^{2}}{e^{2}}\right]
$$

and again using $n=(5 r / 2)^{6 / 5}$ but the transformation $e=\sin \varphi \sqrt{1+\cos ^{2} \varphi}$ leads to the metric

$$
\mathrm{d} s^{2}=\mathrm{d} r^{2}+\frac{r^{2}}{c_{t}^{2}}\left[\mathrm{~d} \varphi^{2}+\frac{\sin ^{2} \varphi\left(2-\sin ^{2} \varphi\right)^{2}}{4 \cos ^{4} \varphi} \mathrm{d} \theta^{2}\right]
$$

where $c_{t}=2 / 5<c<1$. As before, restricting to $\mathbf{S}^{2}$ leads to the metric $\mathrm{d} \varphi^{2}+m(\varphi) \mathrm{d} \theta^{2}$ where

$$
m(\varphi)=\frac{\sin ^{2} \varphi\left(2-\sin ^{2} \varphi\right)^{2}}{4 \cos ^{4} \varphi}
$$

This corresponds in the case $\lambda=1$ to the deformation of the round sphere using the homotopy $m_{\lambda}(\varphi)=$ $X R(\lambda X), X=\sin ^{2} \varphi$ and

$$
R(X)=\frac{(1-X / 2)^{2}}{(1-X)^{2}}=\frac{1}{4}\left(1+\frac{2}{1-X}+\frac{1}{(1-X)^{2}}\right) .
$$

In this case the metric is not smooth for $\lambda=1$, since the equator is a pole of order 2 . This singularity is not removable since the Gauss curvature $G \rightarrow-\infty$ when $e \rightarrow 1^{-}$. 
A first task in this article is to parameterize the geodesics for a class of deformations of the round sphere, one consequence being the computation of the period, in order to evaluate the conjugate and cut loci in our case studies. The complexity of the computations is related to the transcendance of the problem and the category of functions needed: harmonic, elliptic or higher. Another motivation is to analyze the convexity properties of the injectivity domain of a point $q_{0}$ defined by

$$
I\left(q_{0}\right)=\left\{t p_{0}, t \in\left[0, t_{\text {cut }}\left(q_{0}, p_{0}\right)\right], H\left(q_{0}, p_{0}\right)=1 / 2\right\}
$$

where $t_{\text {cut }}$ is the cut time along the geodesic emanating from $q_{0}$ with adjoint vector $p_{0}$. The convexity issue plays an important role in the regularity theory of optimal transport maps on Riemannian manifolds (see the monograph [25] for more on optimal transportation) and for surfaces, convexity of all injectivity domains holds for any small enough $C^{4}$-perturbation of the round metric on the sphere [15]. This property is analyzed in details in our cases that are one parameter smooth deformations of the round sphere. In particular our computations allow to analyze the relations between the Gauss curvature, the convexity of the period mapping and the convexity of the injectivity domains.

The organization of this article is the following. In Section 2, we introduce in a Hamiltonian setting the concepts to analyze Riemannian metrics on two-spheres of revolution and we classify the complexity of the computations. We recall the results from [8] relating the computations of the conjugate and cut loci in the reflectional symmetric case with the convexity of the period mapping. Section 3 presents the main results of this article concerning the structure of the conjugate or cut loci, and convexity of the injectivity domains.

\section{Almost-Riemannian metrics on two-Spheres of Revolution}

The objective of this section is to introduce the concepts and to recall the properties of the metrics on two-spheres of revolution. The main references are $[6,8,22]$.

\subsection{Preliminaries}

We shall consider Riemannian metrics on the two-spheres of revolution which can be put in the polar form $g=\mathrm{d} \varphi^{2}+m(\varphi) \mathrm{d} \theta^{2}$ which is the standard covering excluding the poles; $\varphi \in(0, \pi)$ is the angle along a meridian, $\varphi=0$ being the north pole and $\theta$ is the angle of revolution extended to $\theta \in \mathbf{R}$. Many of such metrics can be constructed by restricting the Euclidian metric to a surface of revolution diffeomorphic to $\mathbf{S}^{2}$, an example being the round sphere where $m(\varphi)=\sin ^{2} \varphi$. In all this article we shall assume that $g$ is reflectionally symmetric with respect to the equator: $m(\pi-\varphi)=m(\varphi)$. With applications in mind, our analysis will be devoted to the case $m(\varphi)=X R(X), X=\sin ^{2} \varphi$ and $R(0)=1$. It can be interpreted as a deformation of the round sphere using the homotopy $m_{\lambda}(\varphi)=X R(\lambda X)$. We shall moreover assume that $m^{\prime}(\varphi) \neq 0, \varphi \in(0, \pi / 2)$. The Gauss curvature is given by

$$
G=-\frac{1}{\sqrt{m}} \frac{\partial^{2} \sqrt{m}}{\partial \varphi^{2}}
$$

and we shall restrict to the case when the Gauss curvature admits an extremum on the equator. In our study the Riemannian situation will be extended to the almost-Riemannian case where the mapping has a pole at the equator. It will be called the Grušin case if the pole is of order one and the tangential case if the pole is of order two (see $[1,11]$ for the analysis of such metrics).

\subsection{Properties of the geodesic and integration}

Reference [4] is a general introduction to the metrics on surfaces of revolution. We use below the Hamiltonian formalism that withstands such generalizations as almost-Riemannian metrics. A remarkable property is the simplicity of the geodesic flow. Introducing $\psi:=\pi / 2-\varphi$, the Hamiltonian writes

$$
H=\frac{1}{2}\left(p_{\psi}^{2}+\frac{p_{\theta}^{2}}{m(\pi / 2-\psi)}\right)
$$


Parameterizing by arc length $(H=1 / 2)$, this leads to analyze the mechanical system

$$
\left(\frac{\mathrm{d} \psi}{\mathrm{d} t}\right)^{2}+V(\psi)=1, \quad V(\psi)=\frac{p_{\theta}^{2}}{m(\pi / 2-\psi)}
$$

where $p_{\theta}$ is a constant corresponding to the Clairaut relation and where $V(\psi)$ is a one-parameter family of potentials. Equation (2.1) is called the characteristic equation. To integrate we proceed as follows. Consider first the Riemannian case. If $p_{\theta}=0$, the geodesic curves are meridian circles. If $p_{\theta} \neq 0$, a solution can be a parallel but since $m^{\prime}(\varphi) \neq 0$ for $\varphi \in(0, \pi / 2)$, the only parallel solution is the equator. All the other solutions are identical: The potential increases from $V(0)$ to $+\infty$ and cutting by $H=1 / 2$ defines a $\psi$-periodic solution which is symmetric with respect to the equator and oscillates periodically between $-\psi_{+}<0<\psi_{+}$; the period $T$ and the amplitude both depend only upon $p_{\theta}$. In particular, each solution but the equator cuts the equator and is entirely determined by the branch evaluated on the quarter of period $T / 4$ where $\psi(t) \in\left[0, \psi_{+}\right]$. This branch is solution of the differential equation

$$
\frac{\mathrm{d} \psi}{\mathrm{d} t}=\sqrt{1-V(\psi)}
$$

and the period is given by the integral

$$
\frac{T}{4}=\int_{0}^{\psi_{+}} \frac{\mathrm{d} \psi}{\sqrt{1-V(\psi)}}
$$

which depends on $p_{\theta} \in(0, \sqrt{m(\pi / 2)}]$. The application $p_{\theta} \mapsto T\left(p_{\theta}\right)$ is the period mapping. The same is true in the limit case where $m$ has a pole on the equator, $m(\pi / 2)=+\infty$, and every geodesic hits the equator perpendicularly.

The geodesic flow is Liouville integrable and the transcendance of the problem is characterized by the transcendance of the period. More precisely, introducing $X=\sin ^{2} \psi$ where $\psi \in(0, \pi / 2)$, one gets:

$$
\int \frac{\mathrm{d} \psi}{\sqrt{1-V(\psi)}}=\int \frac{\mathrm{d} X}{2 \sqrt{X(1-X)(1-V(X))}} .
$$

We have the following classification.

(i) Harmonic case. After simplification the above integral reduces to an integral of the form:

$$
\int \frac{R(X) \mathrm{d} X}{\sqrt{P(X)}}
$$

where $P(X)$ is a polynomial of degree two, with a trivial real root $X=0$ and $R(X)$ is a rational mapping. (ii) Elliptic case. The previous integral now reduces to the same form (2.3) but $P(X)$ is a polynomial of degree four, with a trivial real root $X=0$ and $R(X)$ is a rational mapping.

A standard process from mechanics consists in parameterizing the solutions with respect to the $\psi$-variable. The algorithm is as follows. One can assume $\psi(0)=0$ and $\dot{\psi}(0)>0$; this leads to start with the ascending branch $\psi(t) \in\left[0, \psi_{+}\right]$where $\dot{\psi}(t)=\sqrt{1-V(\psi)}$. On the interval $\left[\psi_{+},-\psi_{+}\right]$one uses the descending branch. Fixing $\theta(0)=0$ using the symmetry of revolution, the successive intersections of $\theta(t)$ with the equator are denoted $\Delta \theta, 2 \Delta \theta$, and so on. By symmetry one can assume that they are even. Using the relations

$$
\begin{gathered}
\frac{\mathrm{d} \theta}{\mathrm{d} t}=\frac{\partial H}{\partial p_{\theta}}=\frac{p_{\theta}}{m(\varphi)}=\frac{V(\psi)}{p_{\theta}}, \\
\frac{\mathrm{d} \psi}{\mathrm{d} t}= \pm \sqrt{1-V(\psi)}, \quad \mathrm{d} t= \pm \frac{\mathrm{d} \psi}{\sqrt{1-V(\psi)}}
\end{gathered}
$$


one sees that

$$
\begin{aligned}
\theta(t) & =(2 n-1) \Delta \theta+\int_{0}^{\psi(t)} \frac{V(\psi)}{p_{\theta}}\left(-\frac{\mathrm{d} \psi}{\sqrt{1-V(\psi)}}\right) \\
& =(2 n-1) \Delta \theta+\int_{\psi(t)}^{0} \frac{V(\psi) \mathrm{d} \psi}{p_{\theta} \sqrt{1-V(\psi)}},
\end{aligned}
$$

where $n \in \mathbf{N}$ counts the number of intersections with the equator. This computation is also valid in the singular case and this leads to the next definition.

Definition 2.1. The first return mapping to the equator is the smooth mapping $\Delta \theta: p_{\theta} \in(0, \sqrt{m(\pi / 2)}) \mapsto$ $\Delta \theta\left(p_{\theta}\right)$.

\subsection{The optimality problem}

In the previous sections, we have shown that, under the assumption that $m^{\prime}(\varphi) \neq 0$ for $\varphi \in(0, \pi / 2)$, the geodesic flow is especially simple since we have roughly one type of trajectories. For the optimality problem, the situation is more complex since according to Riemannian geometry we have to compute the intersections of geodesics starting from a given point. This leads to introduce the following subset of the cut locus of a given point. The separating locus $L\left(q_{0}\right)$ of $q_{0}$ is the set of points where two distinct optimal geodesics emanating from $q_{0}$ intersect with same length. To compute this locus, the assumption $m(\pi-\varphi)=m(\varphi)$ is crucial. Because of symmetries, the result below is clear.

Proposition 2.2. Given $q_{0}=(\theta(0)=0, \varphi(0))$ and $p_{\theta} \neq 0$, if $\dot{\varphi}(0)=p_{\varphi}(0) \neq 0$, there exist two distinct geodesics intersecting with equal length on the antipodal parallel $\varphi=\pi-\varphi(0)$ and they are associated respectively with $\left(p_{\theta}, \pm \dot{\varphi}(0)\right)$. Similarly, given $q_{0}=(\theta(0)=0, \varphi(0))$ and $p_{\theta} \neq 0$, there exist two distinct geodesics associated respectively with $\pm p_{\theta}$ and the same $\dot{\varphi}(0)$ which intersect with equal length on the opposite meridian $\theta=\pi$.

We recall the results of [8]. In order to compute the cut locus the important point is to control the intersection of the geodesics in the north hemisphere.

Theorem 2.3. Consider a metric on a two-sphere of revolution $\mathrm{d} \varphi^{2}+m(\varphi) \mathrm{d} \theta^{2}$, with $m^{\prime}(\varphi)$ nonzero on $(0, \pi / 2)$, $m(\pi-\varphi)=m(\varphi)$. If the first return mapping to the equator is monotone non increasing, the cut locus from a point different from a pole is a subset of the antipodal parallel.

The computation of the conjugate locus of a point different from a pole is a more complicated task. It is based on the work of [22] which uses the fact that the Jacobi equation is integrable and in particular the Jacobi fields can be estimated. The following proposition holds.

Proposition 2.4. Assume that the first return mapping to the equator is monotone non increasing, then the first conjugate time is given by

$$
\frac{\partial \theta}{\partial p_{\theta}}\left(\varphi, p_{\theta}\right)=0
$$

where $\theta$ is parameterized by $\varphi$ according to

$$
\theta\left(\varphi, p_{\theta}\right)=\Delta \theta\left(p_{\theta}\right)-\int_{\psi_{+}}^{\psi} \frac{V(\psi) \mathrm{d} \psi}{p_{\theta} \sqrt{1-V(\psi)}}
$$

(the first conjugate time being between $T / 2$ and $T / 2+T / 4$ ).

Moreover, 
Theorem 2.5. Assume that the first return mapping is such that $\Delta \theta^{\prime}\left(p_{\theta}\right)<0 \leq \Delta \theta^{\prime \prime}\left(p_{\theta}\right)$ on $(0, m(\pi / 2))$ then: (i) The cut locus of a point not a pole is a segment $[\pi-\alpha, \pi+\alpha]$ of the antipodal parallel; (ii) The conjugate locus of a point not a pole has exactly four cusps.

To conclude, a simple relation links the return mapping to the period mapping [8].

\section{Lemma 2.6.}

$$
\frac{\Delta \theta^{\prime}\left(p_{\theta}\right)}{2}=\frac{T^{\prime}\left(p_{\theta}\right)}{4 p_{\theta}}
$$

A remarkable feature is that most of the analysis in the Riemannian case can be generalized to the almostRiemannian one up to a singularity resolution of the problem near the equator. We have the following two results.

Theorem $2.7([6])$. Consider a singular metric $g=\mathrm{d} \varphi^{2}+m(\varphi) \mathrm{d} \theta^{2}$, where $m(\pi-\varphi)=m(\varphi), m^{\prime}(\varphi) \neq 0$, $\varphi \in(0, \pi / 2)$, with a pole located at the equator. Assume that the first return to the equator is monotone non increasing, then the cut loci are antipodal subarcs. The cut locus of a pole is the opposite pole, is equal to the equator minus the point for an equatorial point and to a proper closed subarc of the antipodal parallel otherwise.

Theorem $2.8([6])$. Consider a singular metric as above and assume that the first return mapping is such that $\Delta \theta^{\prime}<0 \leq \Delta \theta^{\prime \prime}$. Then the conjugate loci are reduced to the opposite pole for poles, a double-heart-shaped set with four meridional cusps for equatorial points and an astroid-shaped set with two meridional and to equatorial cusps otherwise.

From our analysis and [2] we deduce the following proposition.

Proposition 2.9. Consider a Riemannian metric $g=\mathrm{d} \varphi^{2}+m(\varphi) \mathrm{d} \theta^{2}, m(\pi-\varphi)=m(\varphi), m^{\prime}(\varphi) \neq 0, \varphi \in$ $(0, \pi / 2)$ and assume that the first return mapping is monotone non increasing. Then for a point not a pole the distance to the cut locus is the length of the geodesic starting tangentially to the parallel and reaching the antipodal parallel tangentially. Moreover the distance function to the cut locus is monotone non decreasing from the conjugate endpoint to the extremity of the antipodal segment where $\theta=\pi$.

As a subset of the tangent bundle, the injectivity domain is defined as $I\left(q_{0}\right)=\left\{t v, t \in\left[0, t_{\text {cut }}\left(q_{0}, v\right)\right],|v|=1\right\}$ where $t_{\text {cut }}$ is the cut time. It can also be computed as a subset of the cotangent bundle: $I\left(q_{0}\right)=\left\{t p_{0}, t \in\right.$ $\left.\left[0, t_{\text {cut }}\left(q_{0}, p_{0}\right)\right], H\left(q_{0}, p_{0}\right)=1 / 2\right\}$. Consider the case of a Riemannian metric $g=\mathrm{d} \varphi^{2}+m(\varphi) \mathrm{d} \theta^{2}$, symmetric with respect to the equator, $m^{\prime}(\varphi) \neq 0$ on $(0, \pi / 2)$. Assume that the first return mapping to the equator is monotone non increasing, then according to our analysis, the cut time along any geodesic excluding the equator is given by $t_{\text {cut }}\left(q_{0}, p_{0}\right)=T\left(p_{\theta}\right) / 2$ where $T$ is the period mapping. Given $\varphi(0), \theta(0)$ can be set to zero due to the symmetry of revolution, so the level set $H=1 / 2$ is parameterized according to

$$
p_{\theta}=\cos \alpha \sqrt{m_{0}}, \quad p_{\varphi}=\sin \alpha, \quad \alpha \in[0,2 \pi] .
$$

Because of symmetries, convexity has only to be checked on a quarter of the curve $\alpha \in[0, \pi / 2]$ and the boundary of the injectivity domain on the cotangent space is:

$$
\alpha \mapsto \frac{T\left(p_{\theta}\right)}{2}\left(p_{\theta}, p_{\varphi}\right):=\left(c_{1}(\alpha), c_{2}(\alpha)\right) .
$$

It will form, provided $T$ is smooth (which is a consequence of the smoothness of the solutions of differential equation with respect to parameters), a smooth closed curve. According to [24] the convexity property of the injectivity domain is equivalent to a constant sign condition of the curvature given by:

$$
K=\frac{c_{1}^{\prime} c_{2}^{\prime \prime}-c_{1}^{\prime \prime} c_{2}^{\prime}}{\left(c_{1}^{\prime 2}+c_{2}^{\prime 2}\right)^{3 / 2}} .
$$




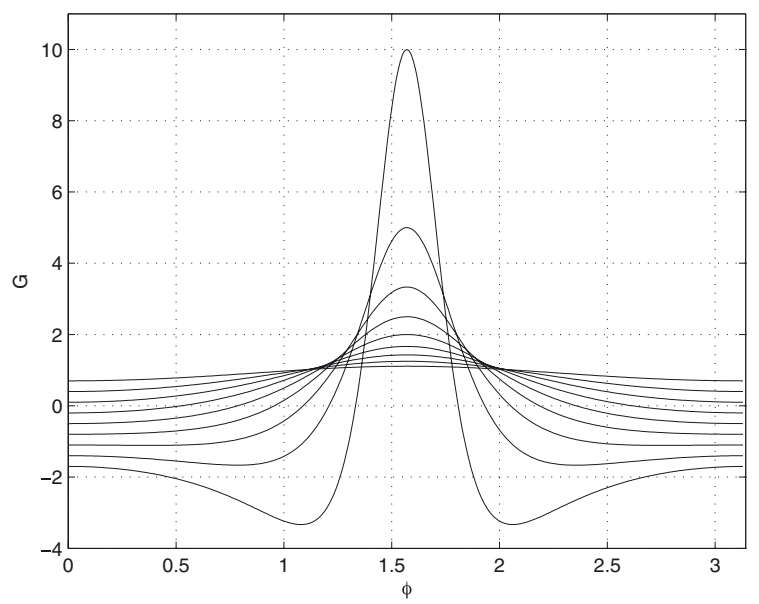

Figure 1. Gauss curvature: variation represented for various $\lambda \in[0,1)$.

In our computation, one shall parameterize the boundary by $p_{\theta}$, restricting $p_{\theta}$ to $\left(0, \sqrt{m_{0}}\right)$; derivatives in $(2.4)$ will be taken with respect to $p_{\theta}\left(\right.$ or $\left.p_{\theta}^{2}\right)$. An advantage of this parameterization is to extend the computation to the singular case, where at the equator $m_{0}=+\infty$. In this case, one restricts $p_{\theta}$ to the convex set $(0,+\infty)$ and the convexity of the injectivity domain will correspond to the convexity with $p_{\theta} \in(0,+\infty)$.

\section{MAIN RESULTS}

According to the previous section our program is to complete the computations of curvature, period mapping and geodesic curves to compute the cut and conjugate loci and to discuss the convexity of the injectivity domains.

\subsection{The harmonic case $m_{\lambda}(\varphi)=\sin ^{2} \varphi /\left(1-\lambda \sin ^{2} \varphi\right), \lambda \in[0,1]$}

As recalled in the introduction, it is associated with coplanar orbit transfers (with two controls) when $\lambda=4 / 5$ and in the limit case $\lambda=1$ it admits a Grušin type singularity at the equator. Note that it was also independently studied in [14] as a perturbation of the round spheres where the geodesic curves are given by elementary functions. The Gauss curvature is (see Fig. 1)

$$
K_{\lambda}=\frac{1}{\left(1-\lambda \sin ^{2} \varphi\right)^{2}}\left((1-\lambda)-2 \lambda \cos ^{2} \varphi\right)
$$

and is strictly negative in the limit case $\lambda=1$. The derivative is given by:

$$
K_{\lambda}^{\prime}=\frac{4 \lambda \sin \varphi \cos \varphi}{\left(1-\lambda \sin ^{2} \varphi\right)^{3}}\left(2(1-\lambda)-\lambda \cos ^{2} \varphi\right) .
$$

For $\lambda \in(0,1)$ it vanishes at the north pole and the equator, $K_{\lambda}$ is monotone for $\lambda \in(0,2 / 3)$ while a local extremum appears when $2=3 \lambda$.

We are in the harmonic case and the characteristic equation reads

$$
\left(\frac{\mathrm{d} \psi}{\mathrm{d} t}\right)^{2}=\frac{\cos ^{2} \psi-p_{\theta}^{2}\left(1-\lambda \cos ^{2} \psi\right)}{\cos ^{2} \psi} .
$$

We denote $Z_{+}$and $Z_{-}$the roots of

$$
1+p_{\theta}^{2}(\lambda-1)=Z^{2}\left(1+\lambda p_{\theta}^{2}\right)
$$


where $Z=\sin \psi$ and the period is given by

$$
\frac{T}{4}=\int_{0}^{Z_{+}} \frac{\mathrm{d} Z}{\sqrt{1+p_{\theta}^{2}(\lambda-1)-Z^{2}\left(1+\lambda p_{\theta}^{2}\right)}} .
$$

Normalizing the amplitude of the oscillation using $Z=Z_{+} Y$ one has

$$
\frac{T}{4}=\int_{0}^{1} \frac{\mathrm{d} Y}{\left(1+\lambda p_{\theta}^{2}\right)^{1 / 2} \sqrt{1-Y^{2}}}=\frac{1}{\sqrt{1+\lambda p_{\theta}^{2}}}[\operatorname{asin} Y]_{0}^{1} .
$$

In particular, one deduces the following [13].

Lemma 3.1. The period is given by

$$
T\left(p_{\theta}\right)=\frac{2 \pi}{\sqrt{1+\lambda p_{\theta}^{2}}}
$$

The $\psi$-variable in the normalized coordinate is given by

$$
\operatorname{asin} Y(t)=\left(1+\lambda p_{\theta}^{2}\right)^{1 / 2} t
$$

This defines a renormalized time $s=\left(1+\lambda p_{\theta}^{2}\right)^{1 / 2} t$. The $\theta$-variable is integrated thanks to the differential equation:

$$
\frac{\mathrm{d} \theta}{\mathrm{d} t}=p_{\theta} \frac{1-\lambda\left(1-\sin ^{2} \psi\right)}{1-\sin ^{2} \psi} .
$$

With the original parameterization this leads to

$$
\theta(t)=\int \frac{p_{\theta} \mathrm{d} t}{\cos ^{2} \psi}-\lambda p_{\theta} t
$$

and finally to

$$
\theta(t)=\frac{p_{\theta}}{\sqrt{1+\lambda p_{\theta}^{2}} \sqrt{1-Z_{+}^{2}}} \operatorname{atan}\left(\sqrt{1-Z_{+}^{2}} \tan \left(t \sqrt{1+\lambda p_{\theta}^{2}}\right)\right)-\lambda p_{\theta} t .
$$

From the period computation we deduce the following.

Theorem 3.2. (i) For $0<\lambda \leq 1$ the first return mapping satisfies $\Delta \theta^{\prime}<0 \leq \Delta \theta^{\prime \prime}$. (ii) For $0 \leq \lambda \leq 1$, all the injectivity domains are convex.

Proof. The period is $T\left(p_{\theta}\right)=2 \pi\left(1+\lambda p_{\theta}^{2}\right)^{-1 / 2}$ so the first assertion is clear. Let $S=\left(1+p_{\theta}^{2}\right)^{-1 / 2}$. Computing, one obtains:

$$
S^{\prime}=-\lambda p_{\theta} S^{3}, \quad S^{\prime \prime}=-\lambda S^{3}\left(1-3 \lambda S^{2} p_{\theta}^{2}\right)^{-1 / 2} .
$$

To compute the boundary of the injectivity domain one introduces the mapping

$$
p_{\theta} \mapsto\left(S p_{\theta}, S p_{\varphi}\right)
$$

and we compute the curvature

$$
K=\frac{c_{1}^{\prime} c_{2}^{\prime \prime}-c_{1}^{\prime \prime} c_{2}^{\prime}}{\left(c_{1}^{\prime 2}+c_{2}^{\prime 2}\right)^{3 / 2}}
$$


Denoting $m_{0}=m(\varphi(0))$, one has

$$
\begin{aligned}
c_{1}^{\prime} & =S^{3}, \\
c_{1}^{\prime \prime} & =-3 \lambda p_{\theta} S^{5}, \\
p_{\varphi}^{\prime} & =-\frac{p_{\theta}}{p_{\varphi} m_{0}} \\
p_{\varphi}^{\prime \prime} & =-\frac{1}{p_{\varphi}^{3} m_{0}}, \\
c_{2}^{\prime} & =-S p_{\theta}\left(\lambda S^{2} p_{\varphi}+\frac{1}{p_{\varphi} m_{0}}\right), \\
c_{2}^{\prime \prime} & =S^{\prime \prime} p_{\varphi}+2 S^{\prime} p_{\varphi}^{\prime}+S p_{\varphi}^{\prime \prime} .
\end{aligned}
$$

From the above computations one deduces

$$
K=-S\left(\frac{\lambda S^{2}}{p_{\varphi}}+\frac{1}{m_{0} p_{\varphi}^{3}}\right)\left(S^{4}+p_{\theta}^{2}\left(\lambda S^{2} p_{\varphi}+\frac{1}{p_{\varphi} m_{0}}\right)^{2}\right)^{-3 / 2}
$$

and $K<0$ for $p_{\theta} \in\left(0, \sqrt{m_{0}}\right)$. When $p_{\theta}=0,\left(c_{1}^{\prime}, c_{2}^{\prime}\right)=(1,0)$ and when $p_{\theta} \rightarrow \sqrt{m_{0}},\left(c_{1}^{\prime}, c_{2}^{\prime}\right) \rightarrow$ $\left(\left(1+\lambda m_{0}\right)^{-1 / 2},-\infty\right)$. This proves the second assertion.

\section{Proposition 3.3.}

(i) In the first quadrant, the boundary of the injectivity domain in polar coordinates $r^{2}=c_{1}^{2}+c_{2}^{2}, \tan \tau=c_{2} / c_{1}$ is given by the oval

$$
r^{2}=\frac{m_{0}}{m_{0}+\left(1+m_{0}(\lambda-1)\right) \cos ^{2} \tau} .
$$

(ii) If $q_{0}$ is on the equator, the curvature is constant and negative and does not depend on $\lambda(K=-1)$.

(iii) The limit of the curvature when $p_{\theta} \rightarrow 0$ and $p_{\theta} \rightarrow m_{0}^{1 / 2}$ does not depend on $\lambda$.

Remark 3.4. In the above computations the crucial point is the relation between the period mapping and its derivative $S^{\prime}=-\lambda p_{\theta} S^{3}$. From the experimental point of view, observe the important variation of the Gauss curvature in Figure 1 (non-monotonicity prevents to apply the results of [23] on the structure of cut and conjugate loci), but the constancy of the curvature of the injectivity domain when $\varphi_{0}=\pi / 2$.

\subsection{The elliptic case}

The remaining computations will be done in elliptic cases using Jacobi or Weierstraß elliptic functions (see $[16,19]$ for general references). Useful formulas are recalled in the appendix. The ellipsoid of revolution is generated by the curve

$$
y=\sin \varphi, \quad z=\varepsilon \cos \varphi
$$

where $0<\varepsilon<1$ corresponds to the oblate case while $\varepsilon>1$ is the prolate one. The restriction of the three dimensional Euclidian metric to the surface is

$$
g=F_{1}(\varphi) \mathrm{d} \varphi^{2}+F_{2}(\varphi) \mathrm{d} \theta^{2}
$$

where $F_{1}=\cos ^{2} \varphi+\varepsilon^{2} \sin \varphi, F_{2}=\sin ^{2} \varphi$, the case $\varepsilon=1$ being the round sphere. The metric can be written in the polar form thanks to

$$
\mathrm{d} \Phi=F_{1}^{1 / 2}(\varphi) \mathrm{d} \varphi
$$


which leads to introduce the elliptic function of the second kind, $\Phi=E(\varphi, \tilde{k})$, with modulus $\tilde{k}^{2}=1-\varepsilon^{2}$. We shall compute the period mapping in the $(\psi, \theta)$-coordinates, $\psi=\pi / 2-\varphi$. The Hamiltonian is

$$
H=\frac{1}{2}\left(\frac{p_{\varphi}^{2}}{F_{1}(\varphi)}+\frac{p_{\theta}^{2}}{F_{2}(\varphi)}\right)
$$

and on $H=1 / 2$, the characteristic equation is

$$
\frac{\mathrm{d} \psi}{\mathrm{d} t}=\frac{\left(\cos ^{2} \psi-p_{\theta}^{2}\right)^{1 / 2}}{\cos \psi\left(\sin ^{2} \psi+\varepsilon^{2} \cos ^{2} \psi\right)^{1 / 2}} .
$$

The roots of $V=1$ in (2.1) are given by $1-p_{\theta}^{2}=\sin ^{2} \psi_{1}$. Making the usual rescaling $Y=\sin \psi_{1} Z$, where $Y=\sin \psi$, the characteristic equation

$$
\frac{\left(Y^{2}+\varepsilon^{2}\left(1-Y^{2}\right)\right)^{1 / 2} \mathrm{~d} Y}{\left(\sin ^{2} \psi_{1}\left(1-Y^{2} / \sin ^{2} \psi_{1}\right)\right)^{1 / 2}}=\mathrm{d} t
$$

becomes

$$
\frac{\left(\varepsilon^{2}+Z^{2} \sin ^{2} \psi_{1}^{2}\left(1-\varepsilon^{2}\right)\right)^{1 / 2} \mathrm{~d} Z}{\left(1-Z^{2}\right)^{1 / 2}}=\mathrm{d} t
$$

Hence the formula for the period mapping is

$$
\frac{T}{4}=\int_{0}^{1} \frac{\left(\varepsilon^{2}+Z^{2} \sin ^{2} \psi_{1}\left(1-\varepsilon^{2}\right)\right)^{1 / 2} \mathrm{~d} Z}{\left(1-Z^{2}\right)^{1 / 2}} .
$$

Introducing

$$
\alpha:=\sqrt{\varepsilon^{2}+\sin ^{2} \psi_{1}\left(1-\varepsilon^{2}\right)}, \quad k^{2}:=\frac{\sin ^{2} \psi_{1}\left(1-\varepsilon^{2}\right)}{\alpha^{2}}, \quad k^{\prime 2}:=\frac{\varepsilon^{2}}{\alpha^{2}},
$$

we get

$$
\begin{aligned}
T & =4 \int_{0}^{1} \frac{\alpha\left(k^{\prime 2}+k^{2} Z^{2}\right) \mathrm{d} Z}{\sqrt{\left(1-Z^{2}\right)\left(k^{\prime 2}+k^{2} Z^{2}\right)}} \\
& =4 \alpha\left[k^{2} K(k)+k^{2} \int_{0}^{1} \frac{Z^{2} \mathrm{~d} Z}{\sqrt{\left(1-Z^{2}\right)\left(k^{\prime 2}+k^{2} Z^{2}\right)}}\right] \\
& =4 \alpha k^{\prime 2} K(k)+4 \alpha k^{2} \int_{0}^{K(k)} \operatorname{cn}^{2} u \mathrm{~d} u
\end{aligned}
$$

with $Z=\operatorname{cn} u$. Using [19],

$$
T=4 \alpha\left(k^{2} K(k)+\left(E(k)-k^{\prime 2} K(k)\right)=4 \alpha E(k) .\right.
$$

Summarizing,

Lemma 3.5. The period maping is $T=4 \alpha E(k), \alpha:=\sqrt{\varepsilon^{2}+\sin ^{2} \psi_{1}\left(1-\varepsilon^{2}\right)}, \sin ^{2} \psi_{1}=1-p_{\theta}^{2}$ and the modulus is $k^{2}=1-\varepsilon^{2} / \alpha^{2}$.

Theorem 3.6. The injectivity domain on an oblate ellipsoid of revolution is convex for any point if and only if the ratio $\varepsilon$ between the minor and the major axis is greater or equal to $1 / \sqrt{3}$. 
Before giving a detailed proof of this result (announced in [9]), we use the quadrature with Jacobi functions of Lemma 3.5 to interpretate the $1 / \sqrt{3}$ ratio.

The period mapping is $T=4 \alpha E(k)$ and the derivatives of $c_{1}\left(p_{\theta}\right)=p_{\theta} T, c_{2}\left(p_{\theta}\right)=p_{\varphi} T$ can be computed using the first and second order complete first integrals $K$ and $E$. One has

$$
\frac{\mathrm{d} \alpha}{\mathrm{d} p_{\theta}}=\frac{p_{\theta}\left(\varepsilon^{2}-1\right)}{\alpha}, \quad \frac{\mathrm{d} k}{\mathrm{~d} p_{\theta}}=-\frac{\varepsilon^{2} p_{\theta} \sqrt{1-\varepsilon^{2}}}{\alpha^{3} \sqrt{1-p_{\theta}^{2}}},
$$

from which we deduce:

$$
\begin{aligned}
\frac{1}{4} \frac{\mathrm{d} c_{1}}{\mathrm{~d} p_{\theta}} & =\alpha E+p_{\theta}^{2} \frac{E\left(\varepsilon^{2}-1\right)}{\alpha}-\frac{\varepsilon^{2} p_{\theta}^{2}(E-K) \sqrt{1-\varepsilon^{2}}}{k \alpha^{2} \sqrt{1-p_{\theta}^{2}}} \\
\frac{1}{4} \frac{\mathrm{d}^{2} c_{1}}{\mathrm{~d} p_{\theta}^{2}} & =\frac{p_{\theta}}{\alpha\left(1-p_{\theta}^{2}\right)^{2}}\left(K\left(3 \varepsilon^{2}-p_{\theta}^{2} \varepsilon^{2}\right)-E\left(2 \varepsilon^{2} p_{\theta}^{4}-4 \varepsilon^{2} p_{\theta}^{2}-2 p_{\theta}^{4}+5 p_{\theta}^{2}-3\right) .\right.
\end{aligned}
$$

In particular if $p_{\theta} \rightarrow 0$, then $\alpha \rightarrow 1, k^{2} \rightarrow 1-\varepsilon^{2}=k_{0}^{2}$ and

$$
\frac{1}{4} \frac{\mathrm{d} c_{1}}{\mathrm{~d} p_{\theta}} \rightarrow E\left(k_{0}\right)>0
$$

For an equatorial point $\varphi(0)=\pi / 2$, when $p_{\theta} \rightarrow 1, \sin ^{2} \psi_{1}=1-p_{\theta}^{2} \rightarrow 0$ and the modulus $k^{2}=\sin ^{2} \psi_{1}\left(1-\varepsilon^{2}\right) / \alpha^{2}$ tends to zero. From the asymptotics recalled in the appendix, one deduces $E \rightarrow \pi / 2, E-K \sim-\pi k^{2} / 4$. Hence

$$
\begin{aligned}
\alpha E & \rightarrow \frac{\varepsilon \pi}{2}, \\
p_{\theta}^{2} \frac{E}{\alpha}\left(\varepsilon^{2}-1\right) & \rightarrow \frac{\left(\varepsilon^{2}-1\right) \pi}{2 \varepsilon}, \\
-\frac{\varepsilon^{2} p_{\theta}^{2}(E-K) \sqrt{1-\varepsilon^{2}}}{k \alpha^{2} \sqrt{1-p_{\theta}^{2}}} & \rightarrow \frac{\left(1-\varepsilon^{2}\right) \pi}{4 \varepsilon},
\end{aligned}
$$

so

$$
\frac{1}{4} \frac{\mathrm{d} c_{1}}{\mathrm{~d} p_{\theta}} \rightarrow \frac{\left(3 \varepsilon^{2}-1\right) \pi}{4 \varepsilon}
$$

The right-hand side vanishes for $\varepsilon=1 / \sqrt{3}$, which is interpretated as follows: for $\varepsilon$ smaller and close enough to $1 / \sqrt{3}$, the injectivity domain in the quadrant admits a vertical tangent for $\varphi(0)$ close enough from $\pi / 2$; since the boundary is smooth by symmetry, the injectivity domains are not convex. A numerical check suggests that the curvature of the boundary curve $\left(c_{1}, c_{2}\right)$ for $\varphi(0) \in[0, \pi / 2]$ and $\varepsilon<1 / \sqrt{3}$ is negative (see Figs. 2 and 3 ).

We now prove Theorem 3.6, completing the proof sketched in [9]. The proof relies on the use of Weierstraß functions (instead of Jacobi) whose asymptotics provide simple estimates. The curvature condition (2.4) is expressed as a sign condition on the quantity ( with $\left.{ }^{\prime}=\partial / \partial p_{\theta}\right)$

$$
T\left(T+p_{\theta} T^{\prime}\right)+\left(X_{0}-p_{\theta}^{2}\right)\left(2 T^{\prime 2}-T T^{\prime \prime}\right) \geq 0, \quad p_{\theta} \in\left[0, \sqrt{X_{0}}\right]
$$

where $X=\sin ^{2} \varphi$ (and $\left.X_{0}=\sin ^{2} \varphi_{0}\right)$. The period $T\left(p_{\theta}, \lambda\right)$ of the $\varphi$ coordinate is computed using the quadrature in the form of the algebraic curve $\left(X=\sin ^{2} \varphi\right)$

$$
\left(\frac{\dot{X}(\lambda-X)}{\sqrt{\lambda}}\right)^{2}=4\left(X-p_{\theta}^{2}\right)(X-1)(X-\lambda) .
$$

Setting $y=1-p_{\theta}^{2}$ and $x=\lambda-1$, the invariants are

$$
g_{2}(x, y)=\frac{4}{3}\left(x^{2}+x y+y^{2}\right), \quad g_{3}(x, y)=\frac{4}{27}\left(2 x^{3}+3 x^{2} y-3 x y^{2}-2 y^{3}\right),
$$



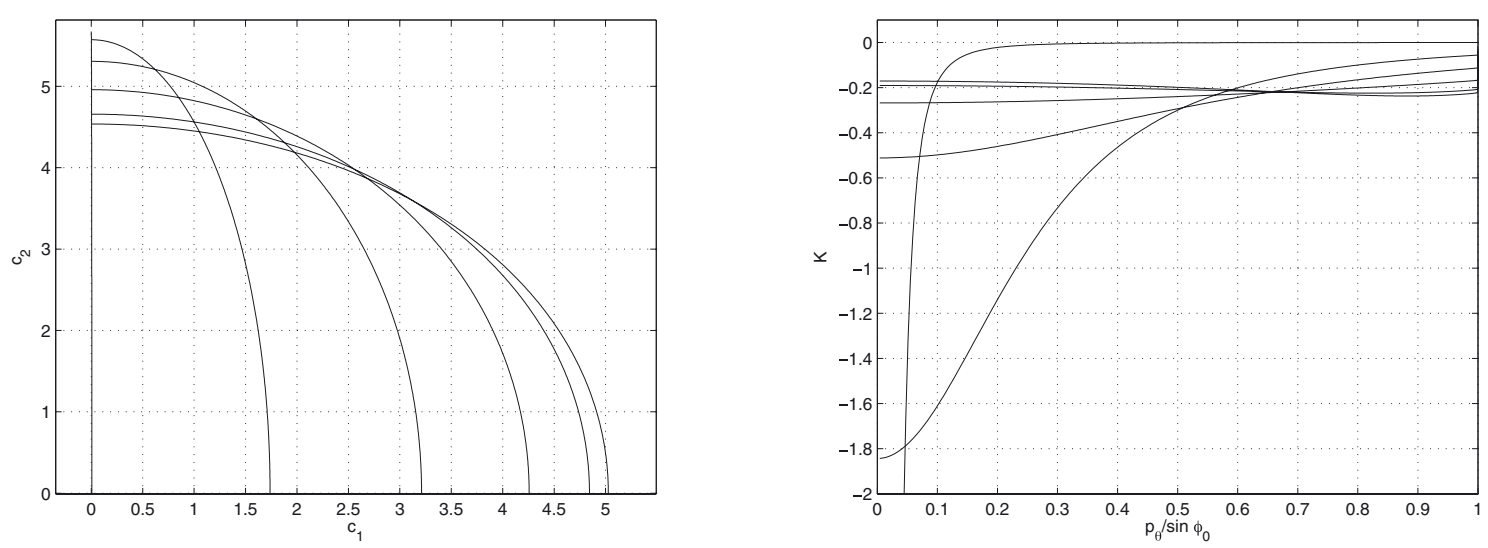

Figure 2. Boundary (on the left) $\left(c_{1}, c_{2}\right)$ of the injectivity domain displayed for the set $\left\{c_{1} \geq 0, c_{2} \geq 0\right\}$ in the case $\varepsilon=0.8>1 / \sqrt{3}$ as well as for several values of $\varphi_{0} \in[0, \pi / 2]$ and its curvature $K$ (on the right), which remains negative, parameterized by $p_{\theta}$.
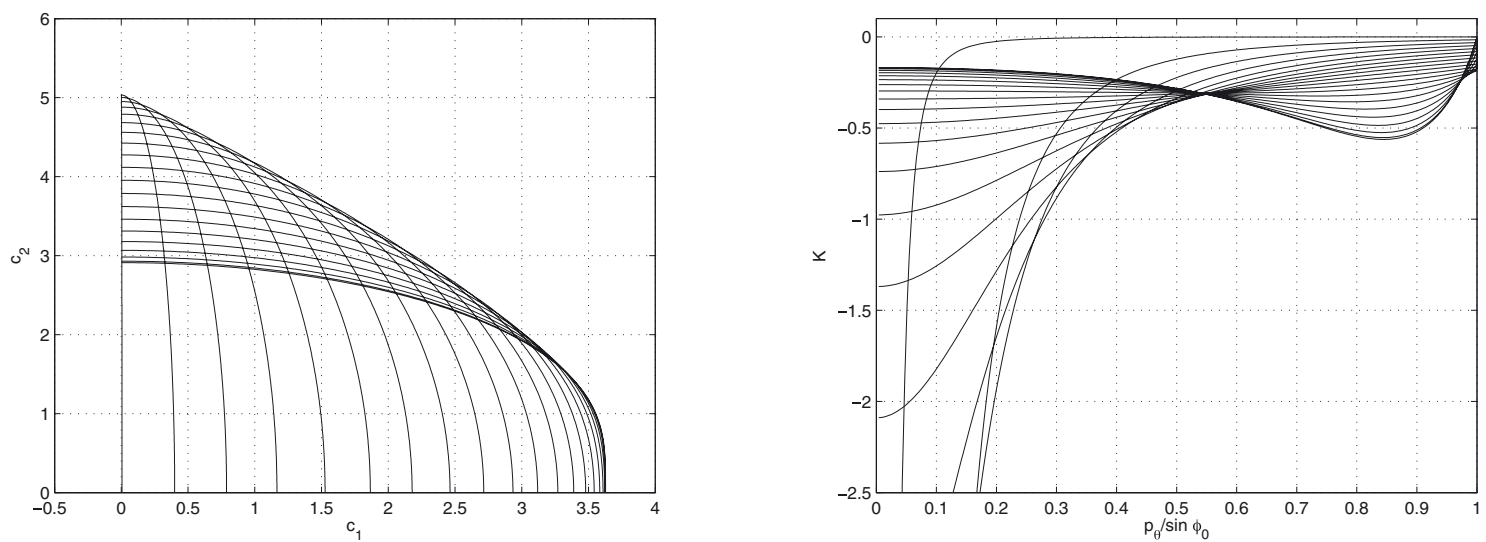

FiguRE 3 . Boundary (on the left) $\left(c_{1}, c_{2}\right)$ of the injectivity domain displayed for the set $\left\{c_{1} \geq\right.$ $\left.0, c_{2} \geq 0\right\}$ in the limit case $\varepsilon=1 / \sqrt{3}$ as well as for several values of $\varphi_{0} \in[0, \pi / 2]$ and its curvature $K$ (on the right), which remains negative, parameterized by $p_{\theta}$.

The period is $T=4 \tau /(3 \sqrt{x+1})$ with

$$
\tau:=(2 x+y) \omega+3 \eta
$$

where $\omega$ is the real half-period of the Weierstraß function associated with $\left(g_{2}, g_{3}\right)$, and $\eta=\zeta(\omega)$. Differentiation with respect to $x$ is obtained through the following rules (see [16] pp. 307-308 for the derivatives of periods or quasi-periods with respect to the invariants)

$$
\delta_{x} \frac{\partial \omega}{\partial x}=-A_{x} \omega-B_{x} \eta, \quad \delta_{x} \frac{\partial \eta}{\partial x}=C_{x} \omega+A_{x} \eta
$$

where

$$
\delta_{x}:=18 x(x+y), \quad A_{x}:=3(2 x+y), \quad B_{x}:=9, \quad C_{x}:=x^{2}+x y+y^{2} .
$$

Symmetrically,

$$
\delta_{y} \frac{\partial \omega}{\partial y}=-A_{y} \omega-B_{y} \eta, \quad \delta_{y} \frac{\partial \eta}{\partial y}=C_{y} \omega+A_{y} \eta,
$$


where

$$
\delta_{y}:=18 y(x+y), \quad A_{y}:=3(x+2 y), \quad B_{y}:=-9, \quad C_{y}:=-\left(x^{2}+x y+y^{2}\right) .
$$

Proposition 3.7. The first and second order derivatives of $\tau$ with respect to (positive) $p_{\theta}$ are

$$
\begin{gathered}
\tau^{\prime}=-\frac{\sqrt{1-y}}{y}(-(x-y) \omega+3 \eta) \\
\tau^{\prime \prime}=-\frac{\left(-2 x^{2}+x(x-2) y+(2 x+1) y^{2}\right) \omega+3(2 x-(x-1) y) \eta}{y^{2}(x+y)} .
\end{gathered}
$$

Define

$$
\alpha(x, y):=\frac{1}{y^{2}}\left(\chi(x, y)-\frac{x}{3}-\frac{y}{6}\right), \quad \chi:=\frac{\eta}{\omega} .
$$

So as to estimate the curvature sign, one essentially needs to compute directional limits of $\alpha$ at the two degeneracies $(x, y)=(0,0)$ and $(\infty, 0)$.

Lemma 3.8. For positive $x$ and $y, \alpha(x, y)>-1 /(2 y)$.

Proof. It is geometrically clear that the period $T$ (hence $\tau$ ) must be strictly decreasing with $p_{\theta}>0$ on an ellipsoid of revolution with prescribed oblateness ( $x$ is fixed). Then, according to Proposition $3.7,-(x-y)+3 \chi>0$, hence the result on $\alpha$.

Remark 3.9. When $x \rightarrow 0$ (flat ellipsoid), $\chi$ degenerates to the rational value $\lim _{x=0} 3 g_{3} /\left(2 g_{2}\right)=-y / 3$ (see [16], p. 314) so one gets $\alpha(0, y)=-1 /(2 y)$ for positive $y$.

Lemma 3.10. For positive $x, \alpha(x, 0)=-1 /(16 x)$.

Proof. When $y \rightarrow 0$ (equator), $\chi$ degenerates to $\lim _{y=0} 3 g_{3} /\left(2 g_{2}\right)=x / 3$. The differentiation rules imply that

$$
\delta_{y} \frac{\partial \chi}{\partial y}=C_{y}+2 A_{y} \chi+B_{y} \chi^{2}
$$

so, iterating, one obtains

$$
\frac{\partial \chi}{\partial y}(x, 0)=\frac{1}{6}, \quad \frac{\partial^{2} \chi}{\partial y^{2}}(x, 0)=-\frac{1}{8 x}
$$

whence the directional limit for $\alpha$ (order two Taylor-Young).

One can then devise a global coarse estimate of $x \alpha$, for instance the following.

Corollary 3.11. For positive $x$ and $y, x \alpha(x, y)>-1 / 15$.

Remark 3.12. One actually has $x \alpha(x, y)>-1 / 16$ for positive $x$ and $y$.

Lemma 3.13. For positive $y, x \alpha(x, y) \rightarrow-1 / 16, x \rightarrow \infty$.

Proof. Set $\xi=1 / x$. When $\xi \rightarrow 0$ (round case $^{3}$ ), $\xi \chi$ degenerates to the limit at $\xi=0$ of

$$
\xi \frac{3 g_{3}}{2 g_{2}}(1 / \xi, y)=\frac{2+3 y \xi-3 y^{2} \xi^{2}-2 y^{3} \xi^{3}}{6\left(1+y \xi+y^{2} \xi^{2}\right)}
$$

\footnotetext{
${ }^{3}$ The degeneracy $x \rightarrow \infty$ towards the round case is interpretated as follows: All geodesics tend to meridians, so the limit has to be independent of $y=1-p_{\theta}^{2}$, and the computation of $\alpha(x, 0)$ in Lemma 3.10 for the equator already gives the result.
} 
so $(\xi \chi)(0, y)=1 / 3$. Computing as in Lemma 3.10, one obtains

$$
\frac{\partial(\xi \chi)}{\partial \xi}(0, y)=\frac{y}{6}, \quad \frac{\partial^{2}(\xi \chi)}{\partial \xi^{2}}(0, y)=-\frac{y^{2}}{8}
$$

whence the directional limit for

$$
\frac{\alpha}{\xi}=\frac{1}{\xi^{2} y^{2}}\left(\xi \chi-\frac{1}{3}-\frac{y}{6} \xi\right)
$$

A global coarse estimate of $(x+1) \alpha$ is for instance as follows.

Corollary 3.14. For positive $x$ and $y,(x+1) \alpha(x, y)<-1 / 17<0$.

Remark 3.15. One actually has $(x+1) \alpha(x, y)<-1 / 16$ for positive $x$ and $y$.

Proposition 3.16. When $x \in(0,1 / 2)$, the curvature for $\varphi_{0}=\pi / 2$ changes sign.

Proof. For $X_{0}=\sin ^{2} \varphi_{0}=1$, up to some positive factor the curvature reads

$$
\kappa=\tau\left(\tau+p_{\theta} \tau^{\prime}\right)+y\left(2 \tau^{\prime 2}-\tau \tau^{\prime \prime}\right) \geq 0
$$

As

$$
\tau+p_{\theta} \tau^{\prime}=3 \omega\left(\left(x-\frac{1}{2}\right)+(1-\alpha) y+2 \alpha y^{2}\right),
$$

we see using Lemma 3.10 that this term has a negative limit as $y \rightarrow 0$ since

$$
\lim _{y=0} \omega=\lim _{y=0} \frac{\pi}{3} \sqrt{\frac{g_{2}}{2 g_{3}}}=\frac{\pi}{2 \sqrt{x}}>0
$$

Moreover,

$$
\tau^{\prime}=-\frac{3 \omega}{2} \sqrt{1-y}(1+2 \alpha y)
$$

and

$$
\tau^{\prime \prime}=-\frac{3 \omega}{2(x+y)}(1+(1+4 \alpha) x+2 \alpha(1-x) y),
$$

are both well defined for $y=0$ so $\kappa$ has the sign of $x-1 / 2$ and is negative. Conversely, when $y=1, \tau^{\prime}$ vanishes and $\kappa=\tau\left(\tau-\tau^{\prime \prime}\right)$ with

$$
\tau-\tau_{\mid y=1}^{\prime \prime}=\omega((x+2)+6 \chi)>3 \omega x>0
$$

by virtue of Lemma 3.8. Hence the change of sign.

Proposition 3.17. When $x \geq 1 / 2, \tau^{\prime \prime} \geq 0$.

Proof. Write as in the previous proof

$$
\tau^{\prime \prime}=-\frac{3 \omega}{2(x+y)}(1+(1+4 \alpha) x+2 \alpha(1-x) y),
$$

and notice that, using Corollary 3.11, the term in the brackets is bounded from below according to

$$
(1+x)+2 \alpha x \underbrace{\left(2+y\left(\frac{1}{x}-1\right)\right)}_{\geq 0}>(1+x)-\frac{2}{15}\left(2+y\left(\frac{1}{x}-1\right)\right) \geq \frac{11}{10}
$$

for $x \geq 1 / 2$. 


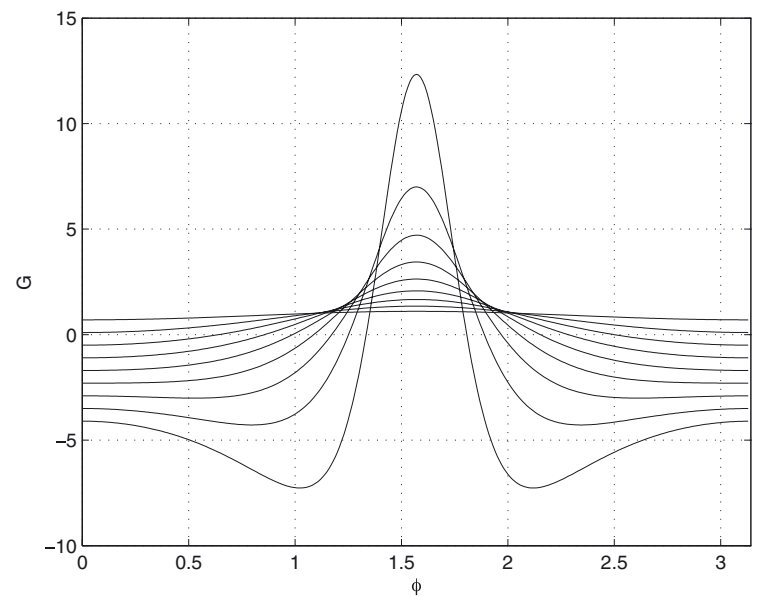

FigURE 4. Gauss curvature: variation for different values of $\lambda \in[0,1]$ (compare with Fig. 1).

Proposition 3.18. When $x \geq 1 / 2, \tau+p_{\theta} \tau^{\prime} \geq 0$.

Proof. Write as in the proof of Proposition 3.16

$$
\tau+p_{\theta} \tau^{\prime}=3 \omega\left(\left(x-\frac{1}{2}\right)+(1-\alpha) y+2 \alpha y^{2}\right),
$$

and notice that, using successively Lemma 3.8 and Corollary 3.14,

$$
(1-\alpha)+2 \alpha y \geq-\alpha>0 .
$$

Proof of Theorem 3.6. When the ratio is less than $1 / \sqrt{3}$, that is when $x<1 / 2$, Proposition 3.16 shows that convexity does not hold for $\varphi_{0}=\pi / 2$. Conversely, when $x \geq 1 / 2$, as $\tau^{\prime \prime} \leq 0$ according to Proposition 3.17, nonnegativeness of

$$
\tau\left(\tau+p_{\theta} \tau^{\prime}\right)+\left(X_{0}-p_{\theta}^{2}\right)\left(2 \tau^{\prime 2}-\tau \tau^{\prime \prime}\right)
$$

holds as soon as $\tau+p_{\theta} \tau^{\prime} \geq 0$, which is Proposition 3.18.

We consider now the case of the deformation

$$
m_{\lambda}(\varphi)=\frac{\sin ^{2} \varphi}{\left(1-\lambda \sin ^{2} \varphi\right)^{2}}
$$

which is a simplification of the situation corresponding to the orbital transfer where the thrust is oriented only in the tangential direction analyzed in the last section. If $\lambda=1$, the equator is a pole of order two, but the period mapping can be evaluated with an integral of the first kind only.

Lemma 3.19. The Gauss curvature is given by (see Fig. 4)

$$
K_{\lambda}=\frac{1-6 \lambda \cos ^{2} \varphi-\lambda^{2} \sin ^{2} \varphi\left(1+\cos ^{2} \varphi\right)}{\left(1-\lambda \sin ^{2} \varphi\right)^{2}} .
$$

One sets again $\psi:=\pi / 2-\varphi$ and we get the characteristic equation

$$
\left(\frac{\mathrm{d} \psi}{\mathrm{d} t}\right)^{2}=-\frac{p_{\theta}^{2} \lambda^{4} \cos ^{4} \psi+\cos ^{2} \psi\left(1+2 \lambda p_{\theta}^{2}\right)-p_{\theta}^{2}}{\cos ^{2} \psi} .
$$


The equation $V=1$ has two real roots, $X_{1}, X_{2}$, whose product is $1 / \lambda^{2}$ :

$$
\begin{aligned}
& X_{1}:=\cos ^{2} \psi_{1}=\frac{\left(1+2 \lambda p_{\theta}^{2}\right)-\sqrt{1+4 \lambda p_{\theta}^{2}}}{2 p_{\theta}^{2} \lambda^{2}}, \\
& X_{2}:=\frac{\left(1+2 \lambda p_{\theta}^{2}\right)+\sqrt{1+4 \lambda p_{\theta}^{2}}}{2 p_{\theta}^{2} \lambda^{2}} .
\end{aligned}
$$

We set $Y=\sin \psi$, hence $X=1-Y^{2}$ and we get the two roots:

$$
\begin{aligned}
& \sin ^{2} \psi_{1}=\frac{2 p_{\theta}^{2} \lambda^{2}-\left(1+2 \lambda p_{\theta}^{2}\right)+\sqrt{1+4 \lambda p_{\theta}^{2}}}{2 p_{\theta}^{2} \lambda^{2}}, \\
& \sin ^{2} \psi_{2}=\frac{2 p_{\theta}^{2} \lambda^{2}-\left(1+2 \lambda p_{\theta}^{2}\right)-\sqrt{1+4 \lambda p_{\theta}^{2}}}{2 p_{\theta}^{2} \lambda^{2}}<0 .
\end{aligned}
$$

Therefore,

$$
\left(\frac{\mathrm{d} \psi}{\mathrm{d} t}\right)^{2}=\frac{p_{\theta}^{2} \lambda^{2}\left(\left(\sin ^{2} \psi_{1}-\sin ^{2} \psi\right)\left(\sin ^{2} \psi-\sin ^{2} \psi_{2}\right)\right)^{1 / 2}}{\cos ^{2} \psi} .
$$

We integrate with the ascending branch and making the rescaling $Y=\sin \psi_{1} Z$, we have:

$$
\frac{\mathrm{d} Z}{p_{\theta} \lambda \sqrt{\left(1-Z^{2}\right)\left(\sin ^{2} \psi_{1} Z^{2}-\sin ^{2} \psi_{2}\right)}}=\mathrm{d} t .
$$

The period mapping is given by:

$$
\frac{T}{4}=\int_{0}^{1} \frac{\mathrm{d} Z}{p_{\theta} \lambda\left(\sin ^{2} \psi_{1}-\sin ^{2} \psi_{2}\right)^{1 / 2} \sqrt{\left(1-Z^{2}\right)\left(k^{2} Z^{2}+k^{\prime 2}\right)}}
$$

where

$$
k^{2}:=\frac{2 p_{\theta}^{2} \lambda^{2}-\left(1+2 \lambda p_{\theta}^{2}\right)+\sqrt{1+4 \lambda p_{\theta}^{2}}}{2 \sqrt{1+4 \lambda p_{\theta}^{2}}}
$$

and $k^{2}+k^{\prime 2}=1$. We deduce

Proposition 3.20. The period mapping is given by $T=4 K(k) / \alpha$, where $\alpha=\left(1+4 \lambda p_{\theta}^{2}\right)^{1 / 4}$ and the modulus is

$$
k^{2}=\frac{2 p_{\theta}^{2} \lambda^{2}-\left(1+2 \lambda p_{\theta}^{2}\right)+\alpha^{2}}{2 \alpha^{2}} .
$$

Remark 3.21. When $p_{\theta} \rightarrow 0$, then $k^{2} \rightarrow 0$. When $\lambda=1$ and $p_{\theta} \rightarrow \sqrt{m(\pi / 2)}=+\infty, k^{2} \rightarrow 1 / 2$. This second estimate is the invariant associated with the pole of order 2 at the equator, computed in the tangential case [11].

The $Z$-variable is

$$
Z(t)=-\operatorname{cn}(K(k)+\alpha t, k)
$$

which leads to the parameterization of $\varphi$ associated with the ascending branch. The $\theta$-variable is found using:

$$
\frac{\mathrm{d} \theta}{\mathrm{d} t}=\lambda(\lambda-2)-\lambda^{2} \sin ^{2} \psi_{1} Z^{2}+\frac{1}{1-\sin ^{2} \psi_{1} Z^{2}} .
$$

Computing using [19] and the elliptic integral of the third kind

$$
\Lambda(u, a, k):=\int_{0}^{u} \frac{\mathrm{d} v}{1-a^{2} \mathrm{sn}^{2} v}
$$

one gets the following (see [13] for a more compact form using Weierstraß functions). 

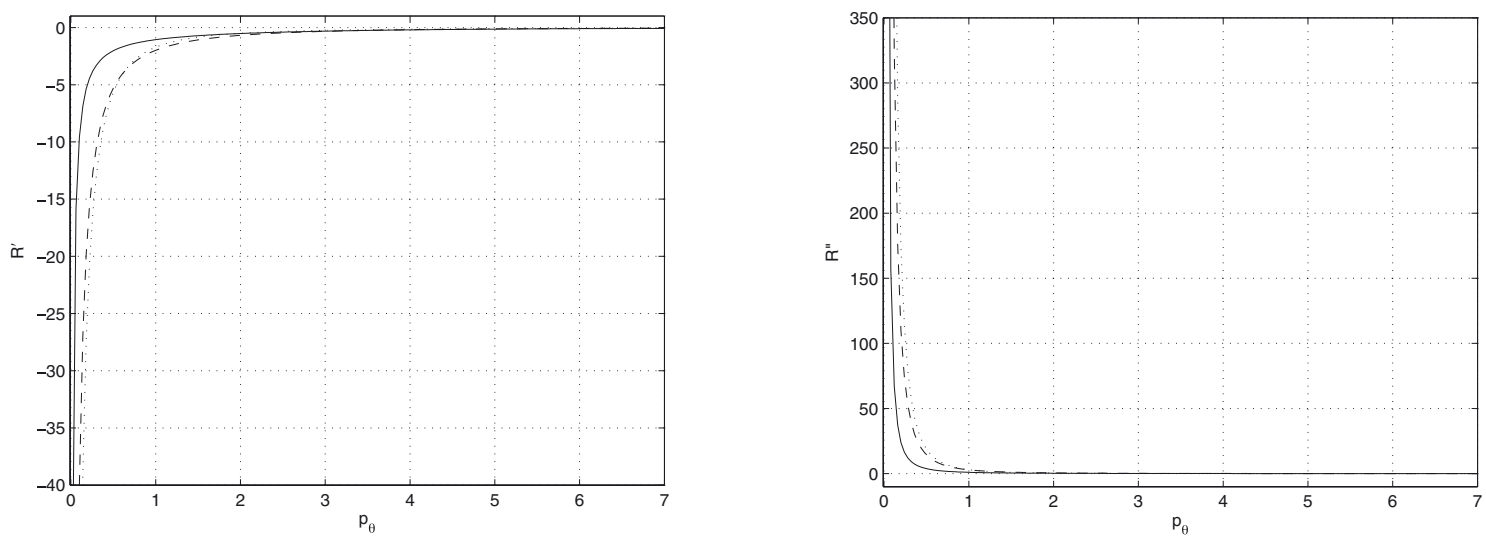

Figure 5. The first and second derivatives of the return mapping with respect to the parameter $p_{\theta}$ are respectively displayed on the left hand side and on the right hand side for different values of $\lambda$, illustrating the fact that $\Delta \theta^{\prime}<0<\Delta \theta^{\prime \prime}(\lambda=0.1$ solid line, $\lambda=0.5$ dash-dot line, $\lambda=0.9$ dotted line).

Proposition 3.22. For the ascending branch, with $\varphi(0)=\pi / 2$ and $\theta(0)=0$, one has:

$$
\begin{aligned}
\varphi(t) & =\frac{\pi}{2}-\operatorname{asin}\left(-\sin \psi_{1} \operatorname{cn}(K+\alpha t, k)\right), \\
\theta(t) & =\theta_{1}(t)+\theta_{2}(t)+\theta_{3}(t),
\end{aligned}
$$

where $\alpha:=\left(1+4 \lambda p_{\theta}^{2}\right)^{1 / 4}$ and

$$
\begin{aligned}
& \theta_{1}(t):=\lambda(\lambda-2) t \\
& \theta_{2}(t):=-\lambda^{2} \sin ^{2} \psi_{1}\left(-\frac{k^{2}}{k^{2}} t+\frac{1}{\alpha k^{2}}(E(K+\alpha t, k)-E)\right), \\
& \theta_{3}(t):=\frac{1}{\alpha \cos ^{2} \psi_{1}}\left[\Lambda\left(K+\alpha t, i \frac{\sin \psi_{1}}{\cos \psi_{1}}, k\right)-\Lambda\left(K, i \frac{\sin \psi_{1}}{\cos \psi_{1}}, k\right)\right] .
\end{aligned}
$$

Elliptic integrals of the third kind are necessary to compute the $\theta$-variable. Thanks to the property that the period mapping is a reparameterization of $K(k)$, one gets estimates of the derivatives which are necessary to conclude about the convexity properties of the first return mapping (see Fig. 5) and the injectivity domains. To compute injectivity domains, one introduces $S:=K(k) / \alpha$ and we denote $c_{1}:=S p_{\theta}, c_{2}:=S p_{\varphi}$. The curvature

$$
K=\frac{c_{1}^{\prime} c_{2}^{\prime \prime}-c_{1}^{\prime \prime} c_{2}^{\prime}}{\left(c_{1}^{\prime 2}+c_{2}^{\prime 2}\right)^{3 / 2}}
$$

can be computed using formal computations and the derivative of $K$ and $E$ with respect to the modulus [19]. One has:

$$
c_{1}^{\prime} c_{2}^{\prime \prime}-c_{1}^{\prime \prime} c_{2}^{\prime}=\frac{\Phi\left(\lambda, p_{\theta}, m_{0}, K, E\right)}{2 \alpha^{8} p_{\theta}^{2} p_{\varphi}\left(-p_{\theta}^{2}+m_{0}\right)\left(p_{\theta}^{2}(\lambda-1)^{2}-1\right)^{2}},
$$

where $\Phi\left(\lambda, p_{\theta}, m_{0}, K, E\right)$ is a polynomial of degree two in $K$ and $E$, and the numerical experiments show that we cannot conclude as for the ellipsoid by a simple verticality argument. The numerical simulations about the curvature lead to the following (see [18] for the details).

Proposition 3.23. There exists $0.7<\lambda_{*}<0.8$ such that for $\lambda \leq \lambda_{*}$ all the injectivity domains are convex but if $\lambda>\lambda_{*}$ there exists $\varphi(0)$ such that the injectivity domain is not convex. 


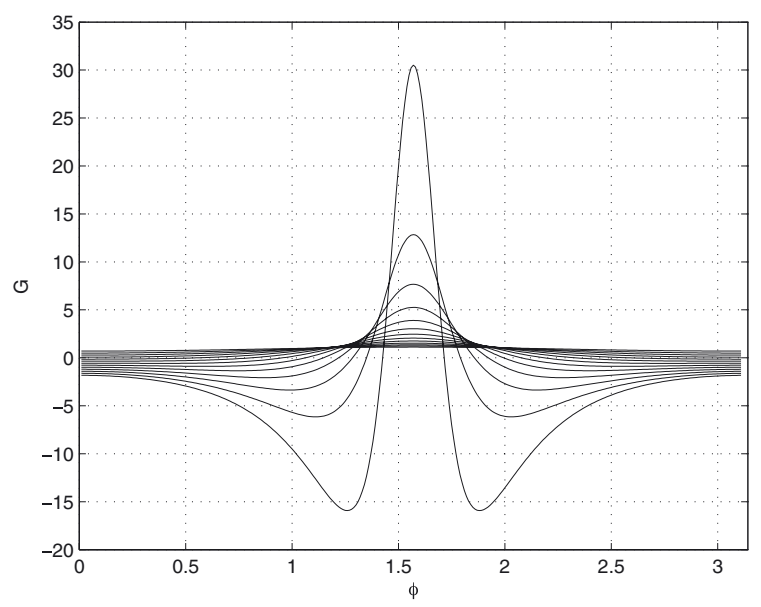

FIGURE 6. Gauss curvature (again, compare with Fig. 1).

In the single-input tangential case, finally, the deformation is

$$
m(\varphi)=\frac{\sin ^{2} \varphi\left(1-\lambda \sin ^{2} \varphi / 2\right)^{2}}{\left(1-\lambda \sin ^{2} \varphi\right)^{2}}
$$

where the period can be computed using general elliptic integrals. We present the algorithms needed in computations in the case $\lambda=1$ only, where the metric admits a pole of order two at the equator.

Lemma 3.24. The Gauss curvature is given by

$$
G=\frac{\left(\sin ^{2} \varphi-4\right)\left(\sin ^{2} \varphi+1\right)}{\left(\sin ^{2} \varphi-1\right)\left(\sin ^{2} \varphi-2\right)}
$$

and $G<0$ outside the equator (see Fig. 6).

Setting $\psi=\pi / 2-\varphi$ and $X=\sin ^{2} \psi$, the characteristic equation $\dot{\psi}^{2}=1-V(\psi)$ is associated with the potential

$$
V(X)=\frac{4 p_{\theta}^{2} X^{2}}{(1-X)(1+X)^{2}}
$$

The equation $1-V(X)=0$ is a polynomial of degree three with three real roots $X_{1}>0>X_{3}>-1>X_{4}$. To integrate, it is sufficient to check that there is no parallel solution $\dot{m}(\varphi)=0$, the equator being excluded since the metric is singular. Meridian circles excepted, every geodesic is such that $\psi$ is periodic with period $T ; T / 4$ is the time to reach from the equator, $X=0$, the root $X_{+}=X_{1}=\sin ^{2} \psi_{+}$,

$$
\frac{T}{4}=\int_{0}^{\psi_{+}} \frac{\mathrm{d} \psi}{\sqrt{1-V(\psi)}}
$$

Since $X=\sin ^{2} \psi$,

$$
\frac{\mathrm{d} t}{2}=\frac{(1+X) \mathrm{d} X}{\sqrt{P(X)}}
$$

where $P(X)=X\left((1-X)(1+X)^{2}-4 p_{\theta}^{2} X^{2}\right)$ is a polynomial whose roots are by construction $X_{1}>X_{2}=0>$ $X_{3}>X_{4}$; so the period is given by

$$
\frac{T}{2}=\int_{0}^{X_{+}} \frac{(1+X) \mathrm{d} X}{\sqrt{P(X)}} .
$$



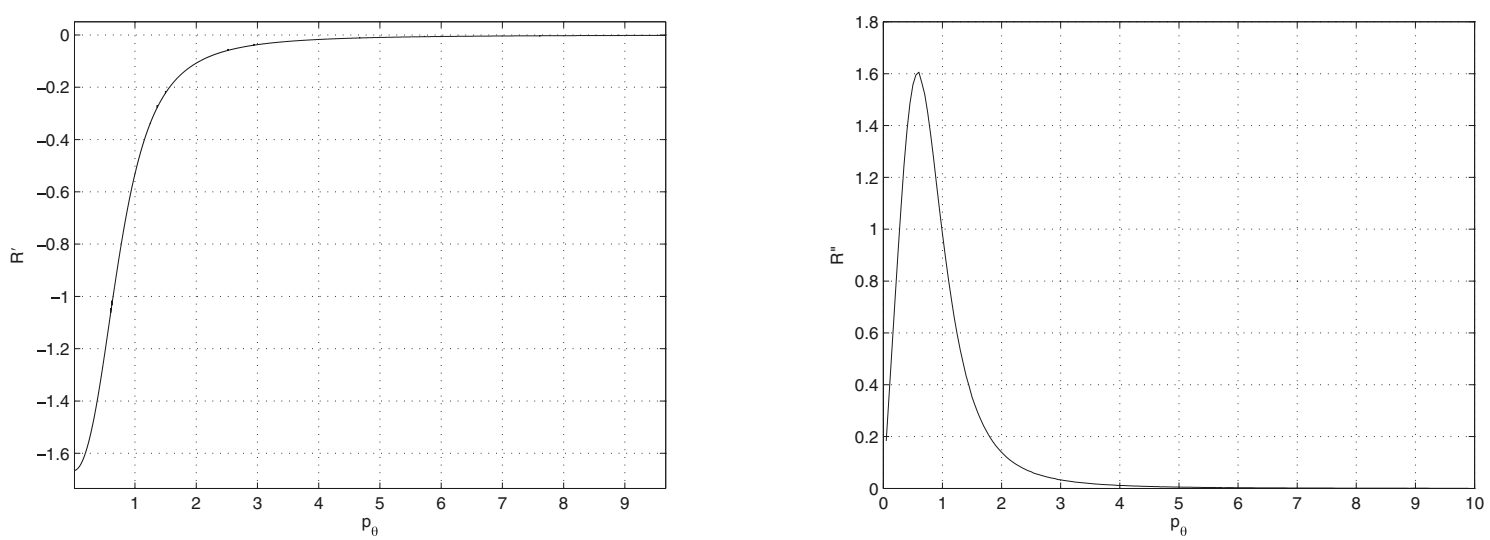

FIGURE 7 . The first and second derivatives of the return mapping with respect to the parameter $p_{\theta}$ are respectively displayed on the left hand side and on the right hand side illustrating the fact that $\Delta \theta^{\prime}<0<\Delta \theta^{\prime \prime}$.

With the rescaling $X=X_{1} Y$ this gives the formula:

$$
T=2 \int_{0}^{1} \frac{\left(1+X_{1} Y\right) X_{1} \mathrm{~d} Y}{\sqrt{P\left(X_{1} Y\right)}} .
$$

The derivatives of $T\left(p_{\theta}\right)$ can be computed in the same category. In particular:

$$
\frac{\mathrm{d} T}{\mathrm{~d} p_{\theta}}=\int_{0}^{1} \frac{X_{1} P_{1}(X 1, Y)+P_{2}\left(X_{1}, Y\right)}{\left(P\left(X_{1} Y\right)\right)^{3 / 2}} \mathrm{~d} Y
$$

where

$$
\begin{aligned}
& P_{1}\left(X_{1}, Y\right):=2\left(1+2 X_{1} Y\right) P\left(X_{1} Y\right)-X_{1} Y\left(1+X_{1} Y\right) P^{\prime}\left(X_{1} Y\right) \\
& P_{2}\left(X_{1}, Y\right):=8 p_{\theta} X_{1}^{4} Y^{3}\left(1+X_{1} Y\right) .
\end{aligned}
$$

The explicit expression of $X_{1}$ is given by Cardano's formula. Due to the complexity, one checks numerically that the first return mapping is such that $\Delta \theta^{\prime}<0<\Delta \theta^{\prime \prime}$ (see Fig. 7). A proof of this is given in [6] using a different parameterization, allowing to conclude on the structure of the cut and conjugate loci.

Remark 3.25. Monotonicity of the return mapping is a consequence of the Gauss curvature condition $G<0$, since conjugate points cannot occur before crossing the equator and geodesics are not intersecting before crossing the equator.

To compute explicitly the period, one starts from

$$
2 \mathrm{~d} t=\frac{(1+X) \mathrm{d} X}{\sqrt{P(X)}}
$$

and makes the reparameterization $2 \mathrm{~d} t=\mathrm{d} s(1+X)$; then $\mathrm{d} s=\mathrm{d} X / \sqrt{P(X)}$ and the $X$-variable is computed in the $s$-time, while the true time is recovered by quadrature. Having reduced the complexity to evaluate

$$
\int \frac{\mathrm{d} X}{\sqrt{P(X)}}
$$


Möbius transformations are used to normalize the roots of the polynomial $P$. A Möbius transformation is a transformation of the Riemann sphere: $X=f(Z)=(a Z+b) /(c Z+d), a d-b c \neq 0$. Such a transformation induces the following on the polynomial: $P(X)=Q(Z) /(c Z+d)^{4}$ where $Q$ is a polynomial of degree four with normalized roots $-1,1,-1 / k$ and $1 / k$. In particular,

$$
\int \frac{\mathrm{d} X}{\sqrt{P(X)}}=\int \frac{(a d-b c)}{\sqrt{Q(Z)}} \mathrm{d} Z
$$

Moreover, the following properties hold. (i) The cross-ratio of four points is invariant:

$$
\frac{\left(X_{1}-X_{3}\right)\left(X_{2}-X_{4}\right)}{\left(X_{2}-X_{3}\right)\left(X_{1}-X_{4}\right)}=\frac{\left(Z_{1}-Z_{3}\right)\left(Z_{2}-Z_{4}\right)}{\left(Z_{2}-Z_{3}\right)\left(Z_{1}-Z_{4}\right)}
$$

(ii) Given two triplets $\left(Z_{1}, Z_{2}, Z_{3}\right)$ and $\left(X_{1}, X_{2}, X_{3}\right)$ of different points, there exists only one Möbius application mapping the two triplets:

$$
F: Z \mapsto \frac{a Z+b}{c Z+d}=X
$$

whose coefficients are

$$
\begin{aligned}
& a:=\left|\begin{array}{lll}
Z_{1} X_{1} & X_{1} & 1 \\
Z_{2} X_{2} & X_{2} & 1 \\
Z_{3} X_{3} & X_{3} & 1
\end{array}\right|, c:=\left|\begin{array}{lll}
Z_{1} X_{1} & 1 \\
Z_{2} & X_{2} & 1 \\
Z_{3} & X_{3} & 1
\end{array}\right|, \\
& b:=\left|\begin{array}{lll}
Z_{1} X_{1} & Z_{1} & X_{1} \\
Z_{2} X_{2} & Z_{2} & X_{2} \\
Z_{3} X_{3} & Z_{3} & X_{3}
\end{array}\right|, d:=\left|\begin{array}{lll}
Z_{1} X_{1} & Z_{1} & 1 \\
Z_{2} X_{2} & Z_{2} & 1 \\
Z_{3} X_{3} & Z_{3} & 1
\end{array}\right| .
\end{aligned}
$$

Different Möbius transformations can be constructed depending on the roots ordering; we choose the one mapping $(1,-1,-1 / k, 1 / k)$ to $\left(X_{1}, X_{2}, X_{3}, X_{4}\right)$ where $k \in(0,1)$ is the modulus. By invariance of the crossratio,

$$
1<\frac{\left(X_{1}-X_{3}\right)\left(X_{2}-X_{4}\right)}{\left(X_{2}-X_{3}\right)\left(X_{1}-X_{4}\right)}=\left(\frac{k+1}{k-1}\right)^{2}
$$

so $k \in(0,1)$. The coefficients of the Möbius transformation are

$$
\begin{gathered}
a:=-X_{1} X_{3}\left(1+\frac{1}{k}\right), \quad b:=a, \\
c:=X_{1}\left(1-\frac{1}{k}\right)-2 X_{3}, \quad d:=-X_{1}\left(1-\frac{1}{k}\right)-\frac{2 X_{3}}{k} .
\end{gathered}
$$

Since this transformation is used to parameterize the branch joining $X_{2}=0$ to $X_{1}$, it has to be checked that the pole of the above Möbius transformation is not in $[-1,1]$ which is numerically done. To parameterize in this case the branch, one uses:

$$
\operatorname{sn}^{-1}(x, k)=\int_{0}^{x} \frac{\mathrm{d} u}{\sqrt{\left(1-u^{2}\right)\left(1-k^{2} u^{2}\right)}} .
$$

This gives the parameterization of the $Z$-variable in the $s$-time, which is a reparameterization of the sn function, while the $X$-variable is obtained inverting the Möbius transformation. To compute the $\theta$-variable, we use

$$
\frac{\mathrm{d} \theta}{\mathrm{d} t}=\frac{4 p_{\theta} X^{2}}{(1-X)(1+X)^{2}}
$$

which can be integrated using elliptic functions. See $[6,18]$ for the details of the computations. 


\section{Conclusion}

We mention to conclude two extensions of our computations motivated by applications to geometry and optimal control.

\subsection{Prolate case}

For the ellipsoid of revolution the prolate case is different: Cut points are not obtained for $T / 2$ (where $T$ is the period mapping) but correspond to geodesics intersecting on the opposite meridian (versus the antipodal parallel in the oblate case). They can be computed solving the equation $\theta=\pi$. This gives an additional complexity when computing the injectivity domains.

\subsection{Extension to mechanical systems on two-spheres of revolution}

They arise for instance in the energy minimization problem for two-level dissipative control systems. This leads to consider mechanical systems where the potential admits several local extrema. See the discussion in [12] for the case of extremals in the meridian planes, and [10] for the non meridian case.

\section{Appendix A. Elliptic integrals And FunCtions}

\section{A.1. Complete elliptic integrals}

The first and second order complete elliptic integrals are respectively defined by

$$
K(k)=\int_{0}^{\pi / 2}\left(1-k^{2} \sin ^{2} \theta\right)^{-1 / 2} \mathrm{~d} \theta, \quad E(k)=\int_{0}^{\pi / 2}\left(1-k^{2} \sin ^{2} \theta\right)^{1 / 2} \mathrm{~d} \theta
$$

where $0<k<1$ is the modulus. One has the following asymptotics when $k \rightarrow 0$ :

$$
K(k)=\frac{\pi}{2}\left(1+\frac{k^{2}}{4}+o\left(k^{3}\right)\right), \quad E(k)=\frac{\pi}{2}\left(1-\frac{k^{2}}{4}+o\left(k^{3}\right)\right) .
$$

\section{A.2. Weierstraß elliptic function}

Weierstraß elliptic function $\wp$ verifies

$$
\wp^{\prime 2}=4 \wp^{3}-g_{2} \wp-g_{3}, \quad \wp(z)-\frac{1}{z^{2}}=\frac{g_{2}}{20} z^{2}+o\left(z^{3}\right)
$$

where $g_{2}$ and $g_{3}$ are called the invariants. Denote $e_{1}, e_{2}, e_{3}$ the complex roots of the cubic polynomial $4 X^{3}-$ $g_{2} X-g_{3}$. Their sum is zero and, if they are real and distinct, they are ordered according to $e_{1}>e_{2}>e_{3}$. In this case, $\wp$ is a doubly periodic function whose half periods $\omega, \omega^{\prime}$ can be chosen real and imaginary, respectively.

\section{REFERENCES}

[1] A. Agrachev, U. Boscain and M. Sigalotti, A Gauss-Bonnet-like formula on two-dimensional almost-Riemannian manifolds. Discrete Contin. Dyn. Syst. 20 (2008) 801-822.

[2] M. Berger, Volume et rayon d'injectivité dans les variétés riemanniennes de dimension 3. Osaka J. Math. 14 (1977) 191-200.

[3] M. Berger, A panoramic view of Riemannian geometry. Springer-Verlag, Berlin (2003).

[4] G. Besson, Géodésiques des surfaces de révolution. Séminaire de Théorie Spectrale et Géométrie S9 (1991) 33-38.

[5] V.G. Boltyanskii, Sufficient conditions for optimality and the justification of the dynamic programming method. SIAM J. Control 4 (1966) 326-361.

[6] B. Bonnard and J.-B. Caillau, Metrics with equatorial singularities on the sphere. HAL preprint No. 00319299 (2008) 1-30.

[7] B. Bonnard and J.-B. Caillau, Geodesic flow of the averaged controlled Kepler equation. Forum Math. 21 (2009) $797-814$.

[8] B. Bonnard, J.-B. Caillau, R. Sinclair and M. Tanaka, Conjugate and cut loci of a two-sphere of revolution with application to optimal control. Ann. Inst. Henri Poincaré, Anal. Non Linéaire 26 (2009) 1081-1098. 
[9] B. Bonnard, J.-B. Caillau and L. Rifford, Convexity of injectivity domains on the ellipsoid of revolution: the oblate case, $C$. R. Acad. Sci. Paris, Sér. I 348 (2010) 1315-1318.

[10] B. Bonnard, J.-B. Caillau and O. Cots, Energy minimization in two-level dissipative quantum control: the integrable case. Proc. of 8th AIMS Conference on Dynamical Systems, Differential Equations and Applications, Dresden (2010). Discrete Contin. Dyn. Syst. suppl. (2011) 229-239.

[11] B. Bonnard, G. Charlot, R. Ghezzi and G. Janin, The sphere and the cut locus at a tangency point in two-dimensional almost-Riemannian geometry. J. Dyn. Control Syst. 17 (2011) 141-161.

[12] B. Bonnard, O. Cots and N. Shcherbakova, Energy minimization problem in two-level dissipative quantum systems. J. Math. Sci. 147 (2012).

[13] J.-B. Caillau, B. Daoud and J. Gergaud, On some Riemannian aspects of two and three-body controlled problems. Recent Advances in Optimization and its Applications in Engineering. Springer (2010) 205-224. Proc. of the 14th Belgium-FrancoGerman conference on Optimization, Leuven (2009).

[14] A. Faridi and E. Schucking, Geodesics and deformed spheres. Proc. Amer. Math. Soc. 100 (1987) 522-525.

[15] A. Figalli, L. Rifford and C. Villani, Nearly round spheres look convex. Amer. J. Math. 134 (2012) $109-139$.

[16] G.-H. Halphen, Traité des fonctions elliptiques et de leurs applications. Première partie, Gauthier-Villars (1886).

[17] J. Itoh and K. Kiyohara, The cut loci and the conjugate loci on ellipsoids. Manuscripta Math. 114 (2004) $247-264$.

[18] G. Janin, Contrôle optimal et applications au transfert d'orbite et à la géométrie presque Riemannienne. Ph.D. thesis, Université de Bourgogne (2010).

[19] D. Lawden, Elliptic functions and applications. Springer-Verlag (1989).

[20] S.B. Myers, Connections between differential geometry and topology I. Simply connected surfaces II. Duke Math. J. 1 (1935) 376-391; 2 (1936) 95-102.

[21] H. Poincaré, Sur les lignes géodésiques des surfaces convexes. Trans. Amer. Math. Soc. 6 (1905) $237-274$.

[22] K. Shiohama, T. Shioya and M. Tanaka, The geometry of total curvature on complete open surfaces. Cambridge University Press (2003).

[23] R. Sinclair and M. Tanaka, The cut locus of a two-sphere of revolution and Toponogov's comparison theorem. Tohoku Math. J. 59 (2007) 379-399.

[24] M. Spivak, A comprehensive introduction to differential geometry II. Publish or Perish (1979).

[25] C. Villani, Optimal transport, Old and new. Springer-Verlag (2009). 